\title{
Imagerie échographique de la glande mammaire de la chèvre en lactation
}

\author{
Moïse HARO ${ }^{1 *}$, Moussa ZONGO ${ }^{1}$, Alidou BAZIE ${ }^{1}$, Wéré PITALA², \\ Drissa S. SANOU ${ }^{1}$ et Raymond BELEMTOUGRI ${ }^{1}$
}
${ }^{1}$ UFR/SVT, Département de Biologie et Physiologie Animales, Laboratoire de Physiologie Animale, Université Ouaga I Pr Joseph Ki-Zerbo, 03 BP 7021 Ouagadougou 03, Burkina Faso. ${ }^{2}$ Ecole Supérieure d'Agronomie, Université de Lomé BP 1515 Lomé, Togo.
*Auteur correspondant; E-mail : haromois2004@yahoo.fr, Tel : +22675265215.

\section{RESUME}

L'échographie est appliquée à la mamelle. L'objectif est d'apprécier l'anatomie interne et le flux de lait des citernes mammaires pendant la lactation chez la chèvre sahélienne. L'étude a porté sur 5 chèvres multipares, d'âge compris entre [36-48] mois et de poids moyen de 32,5 7,3 kilogrammes. Les examens ont été réalisés une fois par semaine pendant cinq semaines. Ils sont pratiqués sur des femelles séparées de leurs petits pendant au moins 12 heures. Le remplissage des citernes a été réalisé à 2, 4, 6 et 8 heures après la tétée. Sur la mamelle rasée, la sonde a été appliquée longitudinalement à l'angle du trayon. Le volume moyen de lait après 12 heures a été de 59,13 $\pm 37,63$ millilitres. Après la tétée le volume moyen de lait résiduel a été de $6,72 \pm 4,16$ millilitres. Le remplissage des citernes au fil du temps après la tétée a été de $9,33 \pm 5,28,15,01 \pm 8,10$, $22,74 \pm 10,54$, et $36,04 \pm 20,41$ millilitres respectivement. La vitesse moyenne de remplissage des citernes mammaires a été de 3,66 $\pm 2,25$ millilitre /heure. La vitesse de remplissage a été plus élevée $(5,26 \pm 1,97$ millilitre /heure) pendant les quatre dernières heures. En somme, l'échographie a permis d'apprécier les flux de lait des citernes mammaires de la chèvre sahélienne en lactation.

(C) 2017 International Formulae Group. All rights reserved.

Mots clés: Chèvre sahélienne, échographie, citerne mammaire.

\section{Ultrasonographic imagery of mammary gland of the goat in lactation}

\section{ABSTRACT}

Ultrasonography is applied to the udder. The objective is to appreciate the internal anatomy and the milk flow of the mammary cisterns during lactation in the sahelian goat. The study related to 5 goats

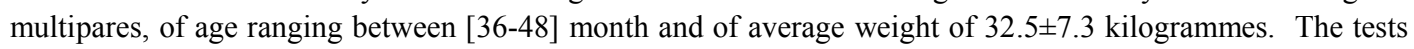
were carried out once per week during five weeks. They were carried out on separate females the small ones during at least 12 hours. The filling of the cisterns was carried out at 2, 4, 6 and 8 hours after suckling. To the shaven udder, the probe was applied longitudinally to the angle of the teat. The average volume of milk after 12 hours was of $59.13 \pm 37.63$ millilitres. After suckling the average volume of residual milk was of $6.72 \pm 4.16$ millilitres. The filling of the cisterns to the wire of time after suckling was of $9.33 \pm 5.28,15.01 \pm 8.10$, $22.74 \pm 10.54$, and $36.04 \pm 20.41$ millilitres respectively. The mean velocity of filling of the mammary cisterns was of 3.66 \pm 2.25 millilitre / hour. The speed of filling was higher (5.26 \pm 1.97 millilitre / hour) during the four 
last hours. All things considered, ultrasonography made it possible to appreciate milk flows of the mammary cisterns of the sahelian goat in lactation.

(C) 2017 International Formulae Group. All rights reserved.

Keywords: Sahelian goat, ultrasonography, mammary cistern.

\section{INTRODUCTION}

La connaissance de l'anatomie interne de la glande mammaire est importante pour un meilleur suivi et une optimisation de la production laitière. Le lait produit dans les alvéoles est ensuite stocké dans les citernes mammaires (Atigui, 2016). L'observation échographique permet d'apprécier les mouvements de lait. (Caja et al., 2004; Buczinski et Descôteaux, 2009). De nombreux travaux ont démontré des différences dans la distribution et l'accumulation du lait des citernes et le lait des alvéoles chez la vache et la brebis (Rovai et al., 2008; Szencziova et Strapak, 2012; Makovický et al., 2015). Chez les mammifères domestiques, les méthodes traditionnelles de visualisation des structures internes de la glande mammaire consiste à la réalisation de coupe anatomique ou par observation échographique de mamelle détachée après abattage de l'animal (Santos et al., 2004; Abshenas et al., 2007; Makovický et al., 2015). Ces méthodes présentent, cependant, des limites liées à la praticabilité et à l'efficacité des tests. Ces dernières années, l'échographie a été appliquée pour examiner la structure et la pathologie mammaires chez les vaches in vivo (Buczinski et Descôteaux, 2009; Esselburn, 2012). Chez la chèvre laitière, des résultats importants ont été rapportés sur les flux de lait dans les citernes au moyen de sondes échographiques (Wojtowski et al., 2006; Fasulkov et al., 2010; Melo et al., 2012). L'observation par échographie de l'organisation interne et du flux de lait dans les citernes mammaires est d'utilité zootechnique importante en clinique, en production animale ou en recherche appliquée (Buczinski et Descôteaux, 2009; Fasulkov, 2012). Les informations sur la santé de la mamelle, l'anatomie et les mouvements de lait permettent de développer les protocoles thérapeutiques les plus appropriés et de suivre les productions laitières dans un troupeau.

Chez la chèvre du sahel, très peu de données existent sur l'anatomie et la fonctionnalité de la glande mammaire. Les travaux de recherche développés sur cette race portent sur la caractérisation de la production laitière en milieu contrôlé ou paysan.

L'objectif de la présente étude est de visualiser, par échographie, la structuration interne de la glande mammaire et les mouvements de lait dans les citernes mammaires pendant la lactation chez la chèvre du sahel. Elle permettra, en outre, d'apprécier le remplissage de la glande mammaire après la tétée.

\section{MATERIEL ET METHODES \\ Animaux et traitements}

Cette étude a été réalisée à la station expérimentale de l'Université Ouaga I Professeur Joseph KI ZERBO, située à Gampéla $\left(12^{\circ} 22^{\prime}\right.$ latitude Nord et $1^{\circ} 31^{\prime}$ longitude Ouest) au Burkina Faso. Elle a porté sur 5 chèvres du Sahel multipares, d'âges et de poids moyens respectifs de $37,25 \pm 1,5$ mois et $32,5 \pm 7,3 \mathrm{~kg}$. Les chèvres ont été séparés en deux lots : un lot à portée simple $(\mathrm{n}=2)$, et un lot à portée double $(\mathrm{n}=3)$. Les animaux ont tous été préalablement contrôlés et déclarés cliniquement indemnes de toute infection parasitaire ou génitale. Ils n'ont pas non plus présenté d'antécédents pathologiques majeurs des fonctions de reproduction et de la lactation avant leur mise en expérimentation. Ils ont ensuite été maintenus dans des conditions standards et uniformes de conduite de l'élevage de la station expérimentale de 
Gampéla. Le troupeau de l'expérience a été gardé en stabulation libre. Leur alimentation a été essentiellement constituée de fourrage vert additionné avec 1 kilogramme par animal et par jour de concentrés alimentaires. Ils avaient un accès libre à l'eau. Toutes les femelles ont été gardées en allaitement avec leurs petits pendant une semaine avant l'expérimentation.

\section{Préparation des animaux}

Avant les observations, les mamelles des chèvres ont été soigneusement lavées à l'eau et les poils rasés à l'aide d'une lame de rasoir. Les mères ont été ensuite séparées de leurs petits toute la nuit, pendant au moins 12h. (Makun et al., 2008). A la suite de cette séparation, la mamelle a été examinée. Les petits ont été allaités ad libitum, puis séparés de leur mère jusqu'au terme des observations $\mathrm{du}$ jour. Les chèvres sont maintenues en station debout dans le couloir à contention, pour l'examen échographique du pis.

\section{Examens échographiques de la mamelle}

Les examens échographiques ont été pratiqués en temps réel en mode $\mathrm{B}$ pour visualiser la structure et estimer les mouvements de lait dans les citernes mammaires. L'échographie a été réalisée à l'aide d'une sonde linéaire de $7,5 \mathrm{MHz}$ et d'une sonde convexe de 3,5 MHz.

Les observations ont été pratiquées une fois par semaine, sur une période de cinq semaines. Elles ont été réalisées sur la mamelle selon la méthode de Ayadi et al. (2003) avec des légères modifications. Elles ont consisté à contrôler le volume de remplissage de la glande mammaire après 12 heures de séparation avec le petit. Une série d'observations ont été ensuite réalisées après la tétée et à intervalle de 2 heures pour contrôler le remplissage des citernes jusqu'à 8 heures après la tétée. Le volume des citernes a été mesuré avant la tétée, juste après la période de tétée $(1 \mathrm{~h})$, et successivement $2 \mathrm{~h}$, $4 \mathrm{~h}, 6 \mathrm{~h}, 8 \mathrm{~h}$ après la tétée.

\section{Procédures d'évaluation du contenu des citernes \\ L'opérateur a d'abord humidifié les} mamelles avec de l'eau. Il a ensuite saisie la base de l'attache des mamelles à l'aide d'une main. Avec l'autre main, la sonde est appliquée longitudinalement au quartier mammaire de sorte à observer le contenu mammaire. D'un mouvement de rotation du poignet, la sonde a été légèrement déviée à l'insertion du trayon. Pour la mamelle gauche, la sonde a été déviée à gauche et à droite pour la mamelle droite. L'opérateur a eu toujours un regard sur l'écran lors des manipulations (Figure 1).

Le contenu des citernes mammaires a été apprécié sur une mamelle pleine dont les petits ont été séparés de la mère $12 \mathrm{~h}$. Après cette séance, la mamelle a été appréciée juste après $1 \mathrm{~h}$ de tétée et ensuite à intervalle de 2 $\mathrm{h}, 4 \mathrm{~h}, 6 \mathrm{~h}$ et $8 \mathrm{~h}$ après la tétée à l'aide d'une sonde linéaire de 7,5 MHz, et parfois avec la sonde convexe de 3,5 MHz. Après avoir gelé l'image sur l'écran, le volume de lait (ml) contenu dans chaque citerne a été mesuré par le même opérateur, à l'aide d'un calliper électronique.

\section{Analyse de données}

Les images ont été collectées sur un support numérique et traitées à l'aide de Microsoft Office Picture Manager 2010. Les résultats ont été exprimés en moyenne \pm écarttype standard. Le volume moyen de lait sécrété a été calculé. La vitesse de remplissage des citernes est considérée comme la quantité de lait obtenue dans la citerne par unité de temps (h). Les traitements graphiques et les valeurs moyennes ont été réalisés à l'aide des logiciels Microsoft Office Excel 2010 et Gen Stat Discovery 2013. Les moyennes ont été comparées à l'aide du test $\mathrm{T}$. Les différences sont considérées comme significatives au seuil de probabilité $\mathrm{p}<0,05$. 


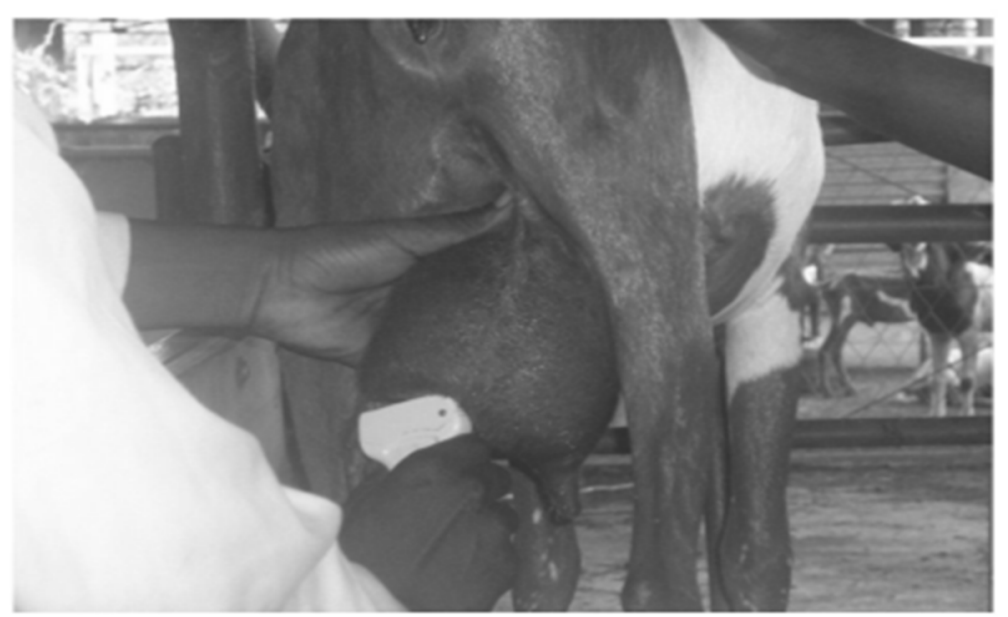

Figure 1 : Examen échographique de la mamelle par contact direct avec la sonde.

\section{RESULTATS}

\section{La structure mammaire}

La coupe échographique des deux mamelles, à l'aide de la sonde convexe de 3,5 $\mathrm{MHz}$, a permis d'observer deux citernes glandulaires de formes irrégulières (Figure 2A). Cet examen des deux mamelles de la chèvre a donné une image de l'anatomie interne de la glande mammaire pleine de lait. La coupe échographique de la glande mammaire en lactation a montré un parenchyme glandulaire dense (échogène) et une citerne glandulaire contenant du lait de forme irrégulière (anéchogène). Un sillon de séparation a été observé entre les deux mamelles. Le parenchyme glandulaire est tapissé de petites zones sombres. L'échographie du trayon plein de lait avec la sonde linéaire de 7,5 MHz a permis d'observé la citerne du trayon (anéchogène), le tissu sous-cutané conjonctif (échogène) et le bout du trayon par lequel le lait est évacué avec la traite ou la tétée (Figure 2B), la peau est apparue hyperéchogène pour la glande et le trayon (Figure 2).

\section{Les mouvements de lait}

Les coupes échographiques prises après la tétée, à $2 \mathrm{~h}, 4 \mathrm{~h}, 6 \mathrm{~h}, 8 \mathrm{~h}$ après la tétée des petits, ont permis d'obtenir les images de citernes de lait anéchogènes à l'aide de la sonde de 7,5 MHz (Figure 3). Ce qui a montré quelques traces de lait après la tétée (Figure 3a) puis le volume de lait a augmenté au fur et à mesure en fonction du temps. Le volume des zones noires (citernes de lait) a été mesuré (Figure 3), la moyenne est obtenue à partir des données des deux citernes. Le lait des citernes est restauré en fonction du temps. Avec la tétée, il y a une vidange du lait des citernes. La sécrétion progressive du lait a été effectuée au fil du temps (Tableau 1).

\section{La vitesse de remplissage des citernes mammaires}

Par la formule ci-dessous, la vitesse de remplissage des citernes de chaque lot de femelle en fonction du temps a été calculée (Tableau 2).

$\mathrm{V}:$ Vitesse

$$
V(m l / h)=\frac{v m(\mathrm{t} 2)-\mathrm{vm}(\mathrm{t} 1)}{\mathrm{t} 2-\mathrm{t} 1}
$$

vm : Volume moyen

t1: Temps 1

t2: Temps 2

La vitesse moyenne de remplissage a été plus élevée $(5,25 \pm 1,97 \mathrm{ml} / \mathrm{h})$ pendant les quatre dernières heures que les quatre premières heures $(2,05 \pm 1,08 \mathrm{ml} / \mathrm{h})$. La vitesse moyenne est évaluée à $3,66 \pm 2,25 \mathrm{ml} / \mathrm{h}$. 


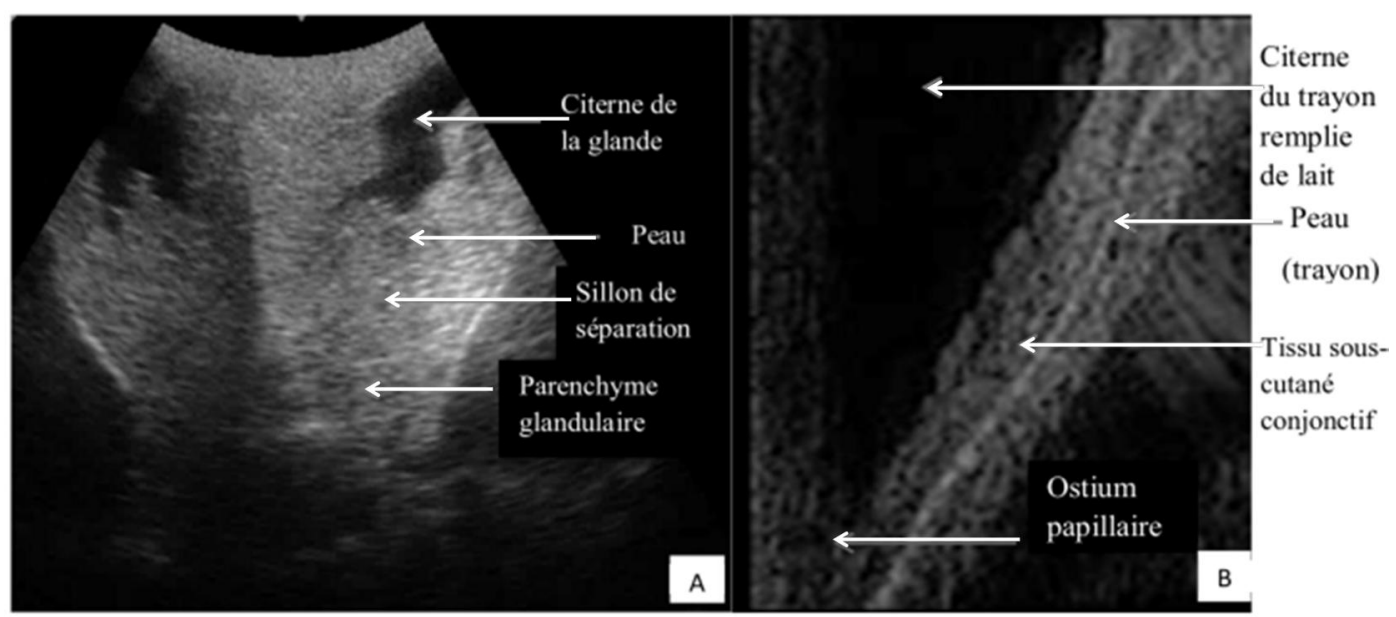

Figure 2 : Anatomie interne des deux glandes mammaires (A) et anatomie interne du trayon (B).
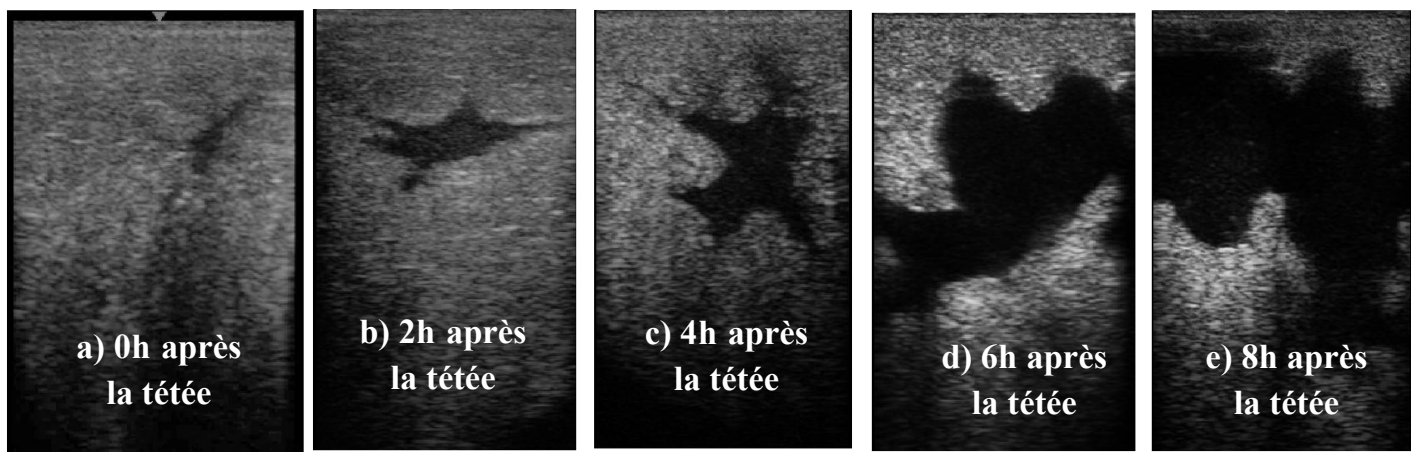

Figure 3 : Evolution du contenu de citerne mammaire après la tétée et $8 \mathrm{~h}$ après la tétée.

Tableau 1 : Cinétique de restauration des citernes mammaires.

\begin{tabular}{lcccccc}
\hline Temps (heure) & $\mathbf{1 2} \mathbf{~ h}$ & Tétée & $\mathbf{2} \mathbf{h}$ & $\mathbf{4} \mathbf{h}$ & $\mathbf{6 ~ h}$ & $\mathbf{8 ~ h}$ \\
\hline $\begin{array}{l}\text { Volume moyen } \\
\text { des citernes (ml) }\end{array}$ & $59,13 \pm 37,63$ & $6,72 \pm 4,16$ & $9,33 \pm 5,28$ & $15,01 \pm 8,10$ & $22,74 \pm 10,54$ & $36,04 \pm 20,41$ \\
\hline
\end{tabular}

Tableau 2 : Vitesse moyenne de remplissage des citernes en fonction du temps.

\begin{tabular}{lc}
\hline Temps (heure) & Vitesse moyenne (ml/h) \\
\hline To & 0 \\
$2 \mathrm{~h}$ & $1,30 \pm 0,56$ \\
$4 \mathrm{~h}$ & $2,84 \pm 1,41$ \\
$6 \mathrm{~h}$ & $3,86 \pm 1.22$ \\
$8 \mathrm{~h}$ & $6,65 \pm 4,93$ \\
\hline
\end{tabular}




\section{DISCUSSION}

La glande mammaire a fait l'objet de nombreuses investigations scientifiques chez différentes espèces de mammifères. En comparant l'anatomie interne de la glande mammaire par rapport à d'autres auteurs, il y a une similitude avec nos résultats (Ayadi et al., 2003; Makovický et al., 2015). De même, l'évolution du volume des citernes, ressemble à l'évolution de la surface des citernes rapportée par Ayadi et al. (2003) à intervalle de 4 h, 8 h, 12 h, 16 h, 20 h, et 24 h, à l'aide de la sonde de $5 \mathrm{MHz}$ chez les vaches. Quant à la sonde de 3,5 Mhz utilisé, il a cette particularité d'effectuer des coupes profondes supérieures ou égale à $15-20 \mathrm{~cm}$. La sonde de 7,5 Mhz a été combinée à la sonde de 3,5 Mhz car cette dernière a présenté ses limites. La sonde de 7,5 Mhz a cette particularité de faire des coupes peu profondes de 5-6 cm. La méthode de Ayadi et al. (2003) modifiée a rapporté pour la première fois sur les mamelles de la chèvre du sahel, des images échographiques de l'anatomie interne. Après une tétée ou une traite, un processus dynamique se produit au niveau de la glande. Il se traduit par l'évolution du volume des citernes en image échographique. La sécrétion du lait au fil du temps traduit la descente du lait alvéolaire vers les citernes pour leur remplissage. Avec la tétée ou la traite, le volume des citernes diminue. Ce phénomène est observé en image échographique montrant que le lait contenu dans la citerne a considérablement diminué. C'est dire qu'il reste toujours du lait dans les alvéoles, il faut attendre un certain temps pour la descente du lait dans les citernes en passant par les canaux galactophores.

Le volume moyen du lait restauré a été estimé à $36,04 \pm 20,41 \mathrm{ml}$ après $8 \mathrm{~h}$ de séparation. Le volume moyen de lait qui a été estimé à $59,13 \pm 37,63 \mathrm{ml}$ après $12 \mathrm{~h}$ de séparation expliquerait la production laitière journalière chez la chèvre du sahel (Makun et al., 2008). Ce résultat montre que le lait est sécrété au fil du temps. En plus, la restauration $\mathrm{du}$ lait varie en fonction $\mathrm{du}$ volume des citernes après la tétée. Plus le volume des citernes est important, plus la restauration $\mathrm{du}$ lait est importante. Ces observations montrent que les femelles laitières peuvent être sélectionnées à partir de la taille de leurs citernes mammaires. Elles correspondent à celles rapportées chez la brebis par Rovai et al. (2008). Ces auteurs ont prouvé qu'il y a une relation entre la taille des citernes et la quantité de lait à traire.

Dans la présente étude, la vitesse moyenne de remplissage des citernes a varié en fonction du temps. La vitesse moyenne obtenue pendant les quatre dernières heures montre que le remplissage des citernes est rapide par rapport aux quatre premières heures.

\section{Conclusion}

Cette étude a rapporté de nouvelles données sur la glande mammaire de la chèvre du sahel qui est encore peu connu. Ces données sont des indices précieux pour le suivi et l'optimisation de la production laitière. La méthodologie utilisée a permis de mettre en évidence la structure interne de la glande mammaire et les mouvements de lait. Cependant, les sondes utilisées n'ont pas permis de visualiser l'activité interne des alvéoles. La technique de l'échographie utilisée est une méthode simple, non invasive, appropriée pour explorer l'anatomie interne des mamelles et d'apprécier les citernes mammaires. Le plateau de lactation pourrait 
être atteint lors des prochaines études. Ces observations échographiques pourraient également contribuer à sélectionner les femelles laitières de chèvre du sahel.

\section{CONFLIT INTERETS}

Les auteurs déclarent qu'ils n'ont aucun conflit d'intérêt pour cet article.

\section{REMERCIEMENTS}

Nous exprimons sincèrement notre gratitude à l'AIEA, à la FIS au FCN/PPAAO et à la coopération belge ARES-CCD pour leurs soutiens en matériels. Nous remercions également l'équipe de travail du Laboratoire de Physiologie Animale pour leur franche collaboration.

\section{REFERENCES}

Abshenas J, Vosough D, Masoudifard M, Molai MM. 2007. B-mode ultrasonography of the udder and teat in camel (Camelus dromedarius). Journal of Veterinary Research, 62(2): 27-31.

Atigui M, Marnet PG, Hager H, Bessalah S, Khorchani T, Hammadi M. 2016. Relationship between external and internal udder and teat measurements of machine milked dromedary camels. Trop Anim Health Prod., 48: 935-942. DOI: 10.1007/s11250-016-1059-91

Ayadi MG, Caja X, Knight CH. 2003. Use of ultrasonography to estimate cistern size and milk storage at different milking intervals in the udder of dairy cows. $J$. Dairy Res., 70: 1-7. DOI: 10.1017/S0022029902005873

Buczinski S, Descôteaux L. 2009. Echographie des bovins. Point Vétérinaire ; 1-191.

Caja G, Ayadi M, Knight CH. 2004. Changes in cisternal compartment based on stage of lactation and time since milk ejection in the udder of dairy cows. J. Dairy Sci., 87(8): 1-7.

Esselburn KM. 2012. Ultrasonographic monitoring of mammary parenchyma growth in preweaned Holstein heifers. $\mathrm{PhD}$ thesis, The Ohio State University, Ohio State, p. 89.

Fasulkov IR, Georgiev PI, Antonov AL, Atanasov AS. 2010. B-mode ultrasonography of mammary glands in goats during the lactation period. Bulgarian Journal of Veterinary Medicine, 13(4): 245-251. DOI: http://tru.unisz.bg/bjvm/BJVM\%20December\%2020 10\%20p.245 251.pdf,

Fasulkov IR. 2012. Ultrasonography of the mammary gland in ruminants: A review. Bulg. J. Vet. Med., 15(1): 1-12.

Makovický P, Margetín M, Milerski M. 2015. Estimation of udder cistern size in dairy ewes by ultrasonography. Mljekarstvo, 65(3): 210-218. DOI: $10.15567 / \mathrm{mljekarstvo.2015.0308}$

Makun HJ, Ajanusi JO, Ehoche OW, Lakpini CAM, Otaru SM. 2008. Growth rates and milk production potential of sahelian and red sokoto breeds goats in northern guinea savannah. Pakistan Journal of Biological Sciences, 11: 601-606. DOI: 10.3923/pjbs.2008.601.606

Melo CHS, Sousa FC, Teles Filho ACA, Moura RR, Albuquerque ES, Pereira AF, Melo LM, Freitas VJF, Teixeira DIA. 2012. Mensurações ultrassonográficas da cisterna da glândula mamária de caprino transgênico [Ultrasound measurements of the mammary gland of transgenic hormone-induced lactating goat]. Arq. Bras. Med. Vet. Zootec., 64(2): 491-494. 
DOI: $\quad$ http://dx.doi.org/10.1590/S010209352012000200033

Rovai M, Caja G, Such X. 2008. Evaluation of udder cisterns and effects on milk yield of dairy ewes. Journal of Dairy Science, 91(12): 4622-4629. DOI: 10.3168/jds.2008-1298

Santos D, Vicente W, Canola J, Léga E. 2004. B-mode ultrasonography in cows during lactation to evaluate the teat anatomy using four different techniques. Brazilian Journal of Veterinary Research and Animal Science, 41(5): 349-354.

Szencziova I, Strapak P. 2012. Ultrasonographiy of the udder and teat in cattle: Perspective measuring technique. Slovak J. Anim. Sci., 45(3): 96-104. DOI: http://www.cvzv.sk/slju/12-3/5Szencziova-SJAS-3-2012review.pdf. Wojtowski J, Slosarz P, Junkuszew A, Milerski M, Szymanowska A, Szymanowski M. 2006. Application of ultrasound technique for cistern size measurement in dairy goats (short communication). Arch. Tierz., Dummerstort., 49(4): 382-388. DOI: http://citeseerx.ist.psu.edu/viewdoc/dow nload?doi $=10.1 \cdot 1 \cdot 600.5334 \&$ rep $=$ rep $1 \& \mathrm{t}$ ype $=$ pdf, 